\title{
Schultze Mechanism
}

National Cancer Institute

\section{Source}

National Cancer Institute. Schultze Mechanism. NCI Thesaurus. Code C92846.

The separation of the placenta from the uterine wall during labor; it begins at the placental center and leads to an expulsion of the placenta after delivery of the baby. 